\title{
Research Article \\ Effect of Surface Error Distribution and Aberration on Electromagnetic Performance of a Reflector Antenna
}

\author{
Binbin Xiang $\mathbb{D}^{1,2}$ Congsi Wang $\mathbb{D}^{1},{ }^{1}$ and Peiyuan Lian ${ }^{1}$ \\ ${ }^{1}$ Key Laboratory of Electronic Equipment Structure Design of Ministry of Education, School of Mechano-Electronic Engineering, \\ Xidian University, Xi'an, Shaanxi 710071, China \\ ${ }^{2}$ Xinjiang Astronomical Observatory, Chinese Academy of Sciences, Urumqi, Xinjiang 830011, China
}

Correspondence should be addressed to Congsi Wang; congsiwang@163.com

Received 12 October 2018; Revised 28 January 2019; Accepted 10 February 2019; Published 2 May 2019

Academic Editor: Claudio Gennarelli

Copyright (C) 2019 Binbin Xiang et al. This is an open access article distributed under the Creative Commons Attribution License, which permits unrestricted use, distribution, and reproduction in any medium, provided the original work is properly cited.

In this paper, a method based on the Zernike distribution and the optical aberration is proposed to investigate the effects of the distribution characteristics of surface distortions of a reflector antenna on its electromagnetic performance (EMP). For large-scale errors, an analytical model is introduced to describe the arbitrary distortions, based on the orthogonal Zernike polynomials. The effects of error distribution described by the Zernike series on typical EMP are analyzed. The numerical results indicate that the distortions in the distribution feature of defocus or spherical aberration have a greater impact on gain, and the distribution feature of tilt or coma mainly influences boresight offset; the distribution feature of defocus, astigmatism, and spherical aberration has a greater impact on sidelobe levels. The results indicate that the beam contour patterns are related to the distribution forms of distortions and are similar with the same aberration feature. On the basis of the Seidel aberration, the relationships between typical EMP and the aberration coefficient are presented. Based on these, the error profile of the primary influence and the approximate feature of EMP can be determined, and the antenna performance can be predicted in a simple manner.

\section{Introduction}

With the development of radio astronomy and deep space exploration, the requirements for the resolution and sensitivity of the reflector antenna are raised. This requires antennas to have a larger aperture size and better electromagnetic performance (EMP) to facilitate obtaining high-quality scientific data $[1,2]$. It is known that the surface quality is one of the key parameters used to evaluate an antenna's mechanical performance $[3,4]$. Reflector surface errors are inevitable, and they may be introduced by manufacturing tolerances or structural deformations caused by environmental loads (e.g., gravity, temperature, wind, and accretions of snow and ice) [5, 6]. Reflector surface errors or aperture phase errors directly influence the antenna gain, boresight pointing, sidelobe level, and beam shape [7]. Many EMP degradations depend on the magnitude, shape, distribution of errors, aperture illumination taper, etc. Hence, understanding the effect of errors on an antenna's EMP is very important.

In general, the mathematical description of the surface error model and the radiation integral can provide a direct connection between structural design methods such as finite element analysis and the resulting EMP of the antenna. However, for convenience, the root mean square (RMS) of surface errors is still currently considered as a significant metric to evaluate the mechanical behavior of antennas [4]. Many studies focus on the determination of the loss in antenna gain from the RMS value. Ruze's formula [8] can be used effectively as a simple estimate and is very critical. However, the gain loss can only be predicted from the knowledge of the RMS of surface errors; other EMP features, such as the pointing offset and sidelobe level, cannot be obtained from the RMS of errors. At present, antenna design demands that the estimates of boresight pointing offset, sidelobe level, and beam pattern deformation must be obtained rapidly and 
accurately. These estimates are very important to evaluate an antenna's overall sensitivity.

Besides Ruze, many other researchers have carried out studies on relevant topics. Rahmat-Samii [9] modified Ruze's formula considering the focal ratio $(F / D)$ and the edge taper:

$$
\Delta G=10 \log \left(e^{-\left(4 \pi k_{g} \varepsilon / \lambda\right)^{2}}\right)
$$

where $\varepsilon$ is the RMS of half of the optical pathlength difference (OPD) and $k_{g}$ is a correction factor that depends on the $F / D$ and the edge taper. In that paper, the effects of random errors on the average power pattern of reflectors were analyzed by using geometrical optics. Corkish [10] presented a survey of the literature relating to the effects of reflector surface distortions on sidelobe levels. The errors are classified on the basis of whether they are random or systematic. Systematic errors are further considered as periodic or nonperiodic errors. Bahadori and Rahmat-Samii [11] studied the influence of periodic and aperiodic surface distortions of cylindrical reflector antennas on the performance of membrane reflector antennas described by a sinusoidal function, such as $\Delta z=\Delta \cdot \sin (2 \pi N x / D)$. Bahadori proposed a closed-form expression to predict some of the unique features of these patterns including the location and the level of the observed grating lobes. Duan and Wang [12] studied the influence of surface random errors and systematic errors on the EMP and established an optimization model for the integrated mechanical-electromagnetic performance. Lian et al. [13] developed a multiobjective optimization model to analyze the effects of surface errors' nonuniformity along the radius on the reflector's radiation characteristic and discussed that for the same RMS of errors for the whole reflector, different distributions along the radius result in different gain losses and sidelobe levels. However, these works consider only one type of error distribution, such as the variations in radial or azimuthal directions, and hence, they cannot represent all situations that influence of the arbitrary error distributions.

For large ground-based reflector antennas, surface distortions are very complicated. With the exception of manufacturing tolerances and misalignment of panels, the backup structure and panels will be deformed due to gravity, temperature, wind, and accretions of snow and ice. Their distribution forms and effects on the EMP are different. The purpose of this paper is to study the effect of different error distribution characteristics from the point of view of the scope of the influence and the aberration. The errors are divided into three types based on different scales of the correlation interval. Zernike polynomials are used to describe wavefront error distributions. These polynomials are completely orthogonal, and their combination can describe arbitrary distortions. The effects of error distributions with the Zernike series on gain loss, boresight pointing offset, and sidelobe level increase are analyzed by geometrical optics. These effects of the Zernike distribution errors are classified and further discussed based on the Seidel aberration. Based on many numerical simulations, the relationships between a typical EMP and the aberration coefficients are presented. We think that the aberration coefficients can be considered as a metric to evaluate the wavefront error behavior. It means that in the presence of wavefront distortions, one can determine the error profile and the approximate feature of the EMP based on the decomposition of the Zernike series and analysis of the aberration. We think that this method is useful for structural design and performance evaluation of a reflector antenna.

\section{Classification in the Correlation Scale for Typical Errors}

Reflector surface distortions can be often defined on the basis of the characteristic correlation interval, $C$, for the phase errors or OPDs (also called wavefront errors) in the aperture plane. It is assumed that the OPD values are completely correlated within a radius of $C$ and completely uncorrelated otherwise $[8,14]$. It is an existent and realistic assumption for segmented surfaces of large ground-based reflector antennas and is a practical way of defining surface irregularity. From the point of view of the correlation interval scale, the distortions can be categorized into three main subdivisions: small-scale errors, intermediate-scale errors, and large-scale errors, shown in Figure 1. For the segmented surface of reflector antennas, the distinction of different scales is related to the aperture diameter, $D$, and length of the reflector panels, $L$. It is assumed as follows:

(a) Small-scale error: $C \leq L$

(b) Medium-scale error: $L<C \leq D / 2$

(c) Large-scale error: $D / 2<C$

If $C \leq L$, the surface irregularity can be distributed in the high spatial frequency form in the aperture surface, as shown in Figure 1(a), and the errors are correlated over areas of the order of the individual panel or less. They may be introduced by manufacturing tolerances, fabrication errors in the reflector panels, and local deformation of the individual panels due to gravity or temperature as well as the errors in the alignment of the panels to the prescribed reflector contour. The small-scale errors are generally considered randomly distributed as panel fabrication and setting errors. The magnitude of errors is usually much less than the wavelength.

If $L<C \leq D / 2$, the surface errors can be distributed in the medium spatial frequency form in an aperture surface, as shown in Figure 1(b), and the errors are correlated over areas of the order of the several panels to half of the circle aperture. They may be caused by local deformation of the backup structure under the influence of local nonuniform temperature force distribution. The time of occurrence and the influence region of the temperature force distribution are random and nonrepeatable as the change of the antenna working case (azimuth and elevation) and environmental conditions. Consequently, the medium-scale errors are generally considered randomly distributed.

If $D / 2 \leq C$, the surface errors can be distributed in the low spatial frequency form in the aperture surface, as shown in Figure 1(c), and the errors are correlated over areas of the 


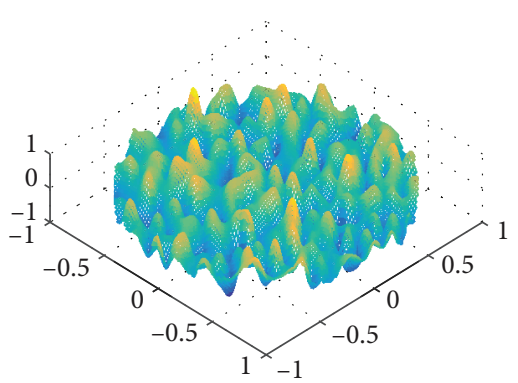

(a) Small-scale errors

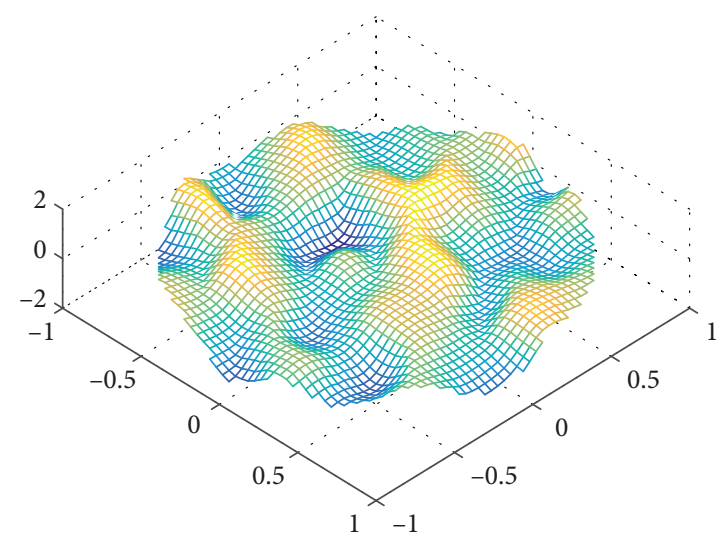

(b) Medium-scale errors

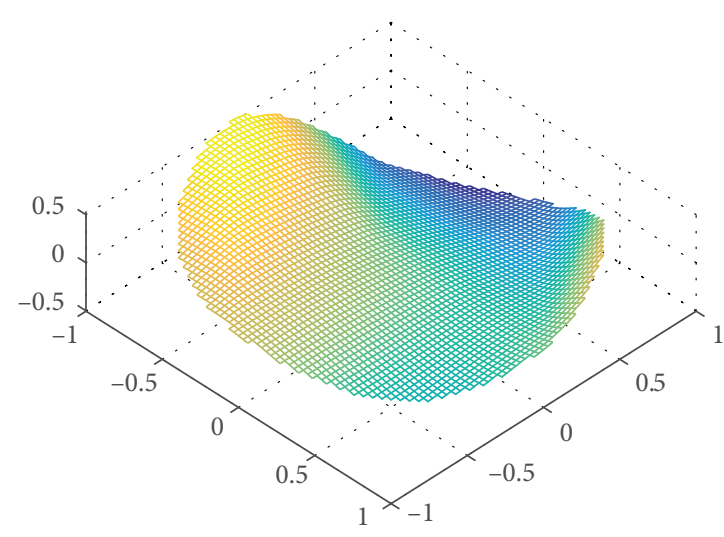

(c) Large-scale errors

FIGURE 1: Reflector antenna surface errors.

order of almost the entire circle aperture. They may be caused by deformation of the backup structure due to gravity or wind. The appearance of wind is usually random, and its effect may be rapid variations. As gravitational loading is constant and its effects are repeatable, these errors are systematic. In this study, large-scale errors only refer to the gravitational deformation of the backup structure and will affect almost the entire reflector surface.

In this paper, the errors will be considered on the basis of whether they are random or systematic, since that is what determines the method of treatment. Unless the random errors of a particular reflector have been characterized by measurement, they must be treated in a probabilistic way to find the most likely effects. Systematic errors can be treated in a deterministic way.

\section{Radiation Formulation}

According to the Fourier transform relationship between the aperture field and the far field, the radiation integral of a standard reflector can be expressed as

$$
\begin{aligned}
E(\psi, \gamma)= & \iint_{A} F(r, \theta) \exp [j k r \sin \psi \cos (\gamma-\theta)] \\
& \cdot \exp (j \Delta \varphi) r d r d \theta,
\end{aligned}
$$

where $F(r, \theta)$ is the aperture field distribution function on the aperture surface $A ; \Delta \varphi$ is the phase error distribution function in the aperture plane caused by surface errors; $(\psi, \gamma)$ are the coordinates of the observation direction

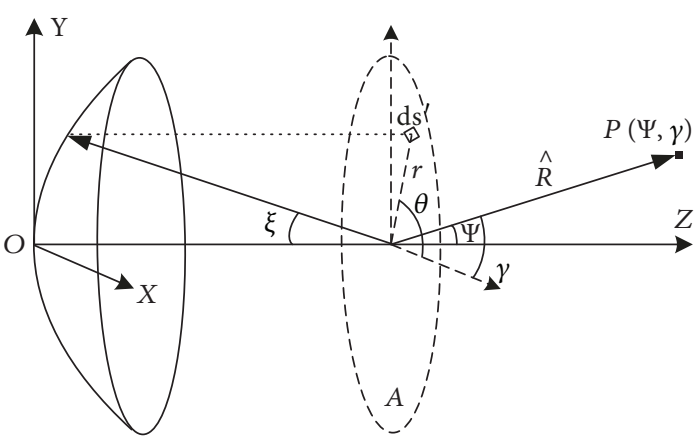

Figure 2: Schematic diagram of the reflector antenna.

in far field; $(r, \theta)$ are the coordinates of the point in the aperture plane; $k$ is the free space wave constant, $k=2 \pi / \lambda$; and $\lambda$ is the working wavelength. The schematic diagram of the reflector antenna is shown in Figure 2.

The phase error is proportional to the OPD and is given by

$$
\Delta \varphi=\delta \cdot k,
$$

where $\delta$ is the OPD caused by surface deformations. The OPD at a point on a reflector surface is greatly exaggerated in Figure 3. It can be seen in Figure 3 that a point $\mathbf{p}_{0}$ on the ideal surface is changed to $\mathbf{p}_{0}{ }^{\prime}$, and the ray pathlength is increased by the sum of the distance of $L 1$ (along the incident ray) and $L 2$ (along the reflected ray) [15]; moreover, the OPD 


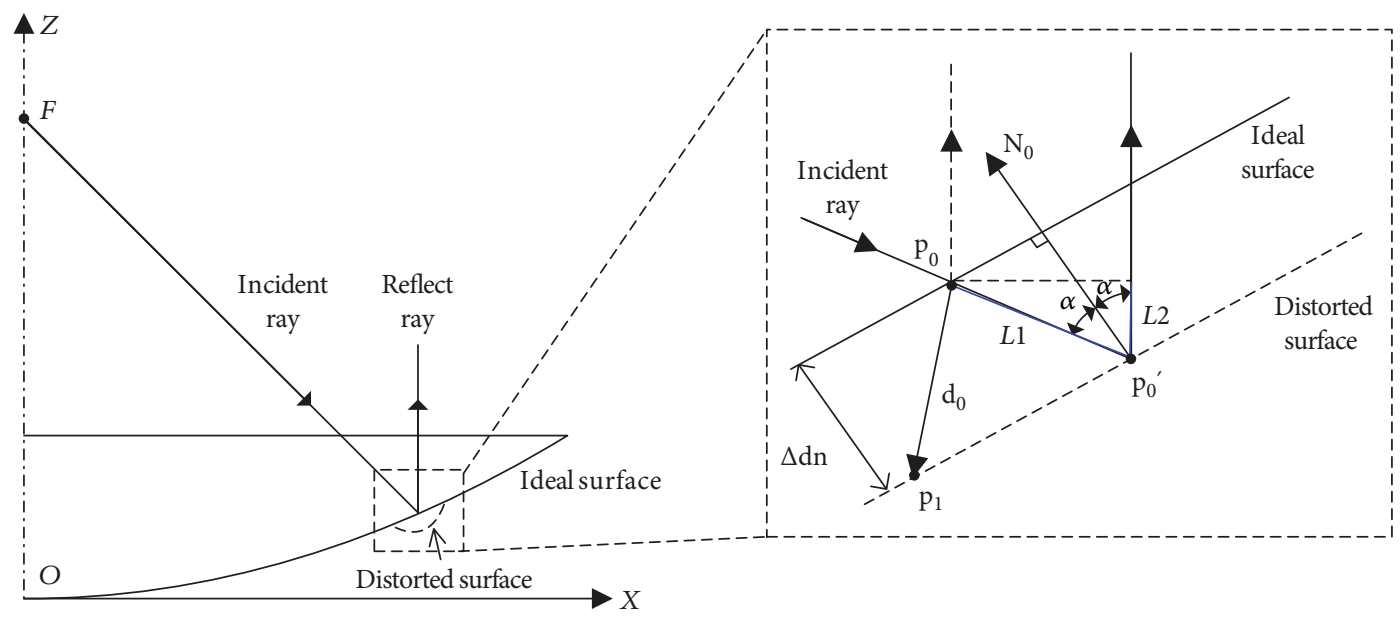

Figure 3: Optical pathlength difference caused by surface deformation.

is equal to twice the axial component of the normal deviation of the surface at that point and is given by

$$
\begin{aligned}
\delta & =L 1+L 2=L 1+L 1 \cdot \cos 2 \alpha \\
& =2 L 1 \cdot \cos ^{2} \alpha \\
& =2 \Delta \mathrm{dn} \cdot \cos \alpha \\
& =2 \mathbf{d}_{0} \cdot \mathbf{N}_{\mathbf{0}} \cdot \cos \alpha,
\end{aligned}
$$

where $\mathbf{d}_{\mathbf{0}}$ is the surface deformation vector, $\mathbf{d}_{\mathbf{0}}=$ $[\Delta d x, \Delta d y, \Delta d z]^{\mathrm{T}} ; \mathbf{N}_{\mathbf{0}}$ is a unit normal vector; and $\Delta \mathrm{dn}$ is the normal component of the deformation vector.

Equation 4 can be used for a primary reflector to compute the OPD for any deformed surface in the normal vector and the axial or normal component of the deformation vector. In this paper, we mainly focus on the descriptions of different scale surface errors and the effects on EM preformation, and the surface error will be expressed in the OPD in general.

In this study, the patterns will be obtained by equation (2) with numerical calculation. In order to verify the validity of EMP results, we analyze the far-field patterns with the numerical calculation and commercial electromagnetic software named GRASP, respectively. The comparison of farfield patterns with radiation integral and GRASP software is shown in Figure 4. We can see that the results from numerical calculation and GRASP software are almost consistent; there are only little differences in null depth and side lobes in the far region. So it indicates that the numerical results are effective and accurate.

\section{Modeling and Analysis of Large-Scale Errors}

The large-scale systematic errors can be described by combination of trigonometric and radial polynomials. It can describe the errors distributed in the radial and azimuthal directions, respectively. However, these polynomial combinations may not be completely orthogonal and cannot accurately describe a complicated distortion, which may be obtained by finite element analysis or measured data.

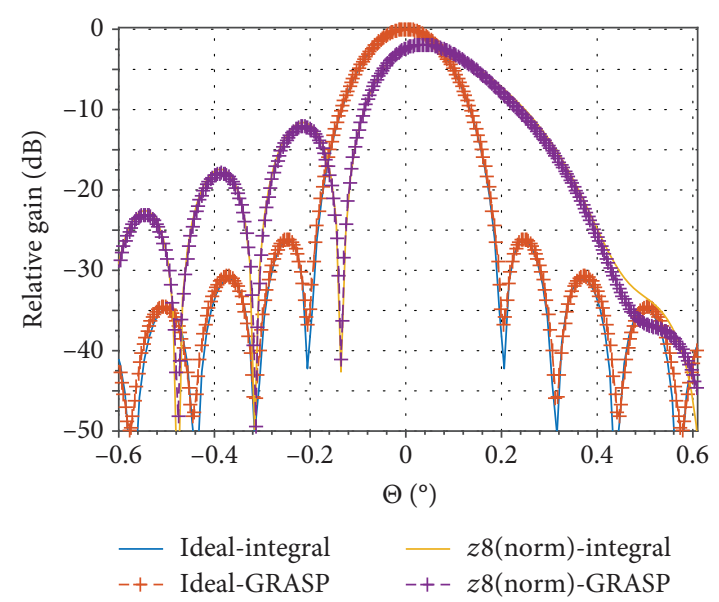

FIGURE 4: Far-field pattern with radiation integral and GRASP software. The curves are the ideal patterns with radiation integral and GRASP software and the distorted patterns with radiation integral and GRASP software, which are caused by optical path difference with the profile of coma (labeled as $z 8$ ).

In general, a set of orthogonal polynomials is needed to model the complicated distortion, and the polynomials have attractive mathematical properties in representing complex surfaces in the form of linear expansion of their basis functions. Recently, almost all researchers in the field of optic telescope have analyzed an arbitrary surface deformation or wavefront/OPD described by a linear combination of a set of orthogonal polynomial series named Zernike polynomials.

4.1. Zernike Polynomial. The Zernike polynomial is a set of complete orthogonal polynomials defined on a unit circle, and the low-level terms correspond with the optical primary aberrations [16-18]. The polynomials are usually expressed in polar coordinates $(r, \theta)$ as products of angular functions and radial polynomials, where $r$ is the normalization radial coordinate ranging from 0 to 1 and $\theta$ is the azimuthal 
TABLE 1: The Zernike circle polynomial sequence modes, orders 1 through 5.

\begin{tabular}{llcccc}
\hline$j$ & $n$ & $m$ & $Z_{j}(r, \theta)$ & $k_{j}$ & Description \\
\hline 1 & 0 & 0 & 1 & 1 & Piston \\
2 & 1 & -1 & $r \sin \theta$ & $1 / 2$ & Vertical tilt \\
3 & 1 & 1 & $r \cos \theta$ & $1 / 2$ & Horizontal tilt \\
4 & 2 & -2 & $r^{2} \sin 2 \theta$ & $1 / \sqrt{6}$ & Oblique astigmatism \\
5 & 2 & 0 & $2 r^{2}-1$ & $1 / \sqrt{3}$ & Defocus \\
6 & 2 & 2 & $r^{2} \cos 2 \theta$ & $1 / \sqrt{6}$ & Horizontal astigmatism \\
7 & 3 & -3 & $r^{3} \sin 3 \theta$ & $1 / \sqrt{8}$ & Vertical trefoil \\
8 & 3 & -1 & $\left(3 r^{3}-2 r\right) \sin \theta$ & $1 / \sqrt{8}$ & Vertical coma \\
9 & 3 & 1 & $\left(3 r^{3}-2 r\right) \cos \theta$ & $1 / \sqrt{8}$ & Horizontal coma \\
10 & 3 & 3 & $r^{3} \cos 3 \theta$ & $1 / \sqrt{8}$ & Oblique trefoil \\
11 & 4 & -4 & $r^{4} \sin 4 \theta$ & $1 / \sqrt{10}$ & Quadrafoil \\
12 & 4 & -2 & $\left(4 r^{4}-3 r^{2}\right) \sin 2 \theta$ & $1 / \sqrt{10}$ & Oblique 2nd-order astigmatism \\
13 & 4 & 0 & $6 r^{4}-6 r^{2}+1$ & $1 / \sqrt{5}$ & Primary spherical \\
14 & 4 & 2 & $\left(4 r^{4}-3 r^{2}\right) \cos 2 \theta$ & $1 / \sqrt{10}$ & Vertical 2nd-order astigmatism \\
15 & 4 & 4 & $r^{4} \cos 4 \theta$ & $1 / \sqrt{10}$ & Quadrafoil \\
16 & 5 & -5 & $r^{5} \sin 5 \theta$ & $1 / \sqrt{12}$ & Pentafoil \\
17 & 5 & -3 & $\left(5 r^{5}-4 r^{3}\right) \sin 3 \theta$ & $1 / \sqrt{12}$ & Pentafoil \\
18 & 5 & -1 & $\left(10 r^{5}-12 r^{3}+3 r\right) \sin \theta$ & $1 / \sqrt{12}$ & $1 / \sqrt{12}$ \\
19 & 5 & 1 & $\left(10 r^{5}-12 r^{3}+3 r\right) \cos \theta$ & $1 / \sqrt{12}$ & 2nd-order coma \\
20 & 5 & 3 & $\left(5 r^{5}-4 r^{3}\right) \cos 3 \theta$ & $1 / \sqrt{12}$ & \\
21 & 5 & 5 & $r^{5} \cos 5 \theta$ &
\end{tabular}

component ranging from 0 to $2 \pi$. In general, the Zernike polynomials are defined as

$$
Z_{j}(r, \theta)=Z_{n}^{m}(r, \theta)= \begin{cases}R_{n}^{\dot{m}^{\prime}}(r) \cos \left(m^{\prime} \theta\right), & \text { if } m \geq 0, \\ R_{n}^{\dot{m}^{\prime}}(r) \sin \left(m^{\prime} \theta\right), & \text { if } m<0,\end{cases}
$$

where the radial function $R_{n}^{\prime}(r)$ is described by

$$
\begin{aligned}
R_{n}^{m}(r) & =R_{n}^{ \pm m^{\prime}}(r) \\
& =\sum_{l=0}^{\left(n-m^{\prime}\right) / 2} \frac{(-1)^{l}(n-l) !}{l !\left[\left(n+m^{\prime}\right) / 2-l\right] !\left[\left(n-m^{\prime}\right) / 2-l\right] !} r^{n-2 l},
\end{aligned}
$$

where the indices $n$ and $m$ are the radial order and the azimuthal frequency, respectively, $m^{\prime}=|m|$, and $j$ is a polynomial number. The values of $n$ and $m^{\prime}$ are always integers and satisfy $m^{\prime} \leq n$ and $n-m^{\prime}=$ even, and the corresponding pairs of $n$ and $m$ are called Zernike modes. Consequently, only polynomials with certain combinations of $n$ and $m$ exist. The first 21 Zernike polynomials, modes, and distribution features are listed in Table 1, and the corresponding shapes of the first 21 polynomials (excluding the first term) are shown in Figure 5.

A significant property of the Zernike polynomials is the orthogonality [17], which is expressed by

$$
\frac{\int_{0}^{1} \int_{0}^{2 \pi} Z_{j}(r, \theta) Z_{j^{\prime}}(r, \theta) r d r d \theta}{\int_{0}^{1} \int_{0}^{2 \pi} r d r d \theta}=\delta_{j j^{\prime}},
$$

where if $j=j^{\prime}, \delta_{j j^{\prime}}=1$, and if $j \neq j^{\prime}, \delta_{j j^{\prime}}=0$. The normalization property of the Zernike polynomials is given by

$$
R_{n}^{m}(1)=1
$$

From Zernike circle polynomials, the associated RMS can be calculated, and the Zernike RMS polynomials 


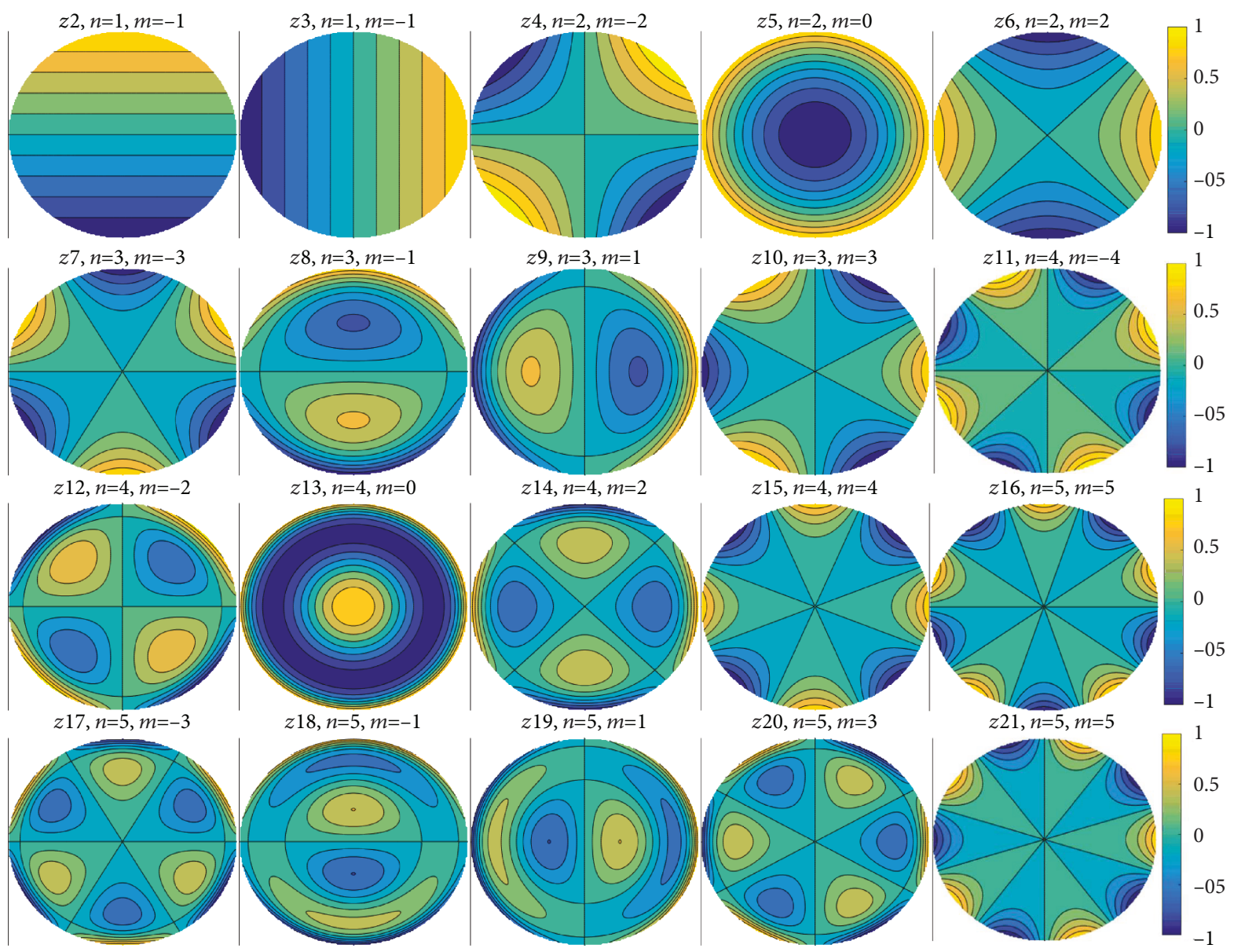

Figure 5: Shape characteristics of the first 21 (excluding the first one) Zernike circle polynomials.

can also be obtained. By integration in a unit circle, the Zernike RMS polynomials are given by

$$
\begin{aligned}
k_{j}\{n, m\} & =\sqrt{\frac{\iint Z_{j}^{2}(r, \theta) r d r d \theta}{A}} \\
& = \begin{cases}1 / \sqrt{n+1}, & \text { if } m=0, \\
1 / \sqrt{2(n+1)}, & \text { if } m \neq 0,\end{cases}
\end{aligned}
$$

where $A$ is the area of a unit circle. The Zernike RMS $k_{j}$ is listed in Table 1 , and $1 / k_{j}$ is usually called the normalization factor.

The reflector surface distortion can be expressed in terms of a sequence of Zernike polynomials in polar coordinates:

$$
\delta(r, \theta)=\sum_{i=1}^{N} a_{i} Z_{i}(r, \theta),
$$

where $a_{i}$ is the coefficient of Zernike polynomials. It is useful to decompose any distortion function (wavefront errors) into components represented by the primary aberrations (tilt, defocus, astigmatism, coma, and spherical aberration), and each polynomial function of the Zernike expansion can provide a good description of each aberration component and extract a component of the wavefront errors. This provides an insight into the mechanical or structural causes of the error.

The RMS of distortion is given by

$$
\sigma=\sqrt{\frac{\iint \delta^{2}(r, \theta) r d r d \theta}{A}}
$$

where $A$ is the area of the antenna's aperture. The RMS value can also be calculated from a vector of Zernike coefficients and the Zernike RMS values as follows:

$$
\begin{aligned}
\sigma^{2} & =\frac{1}{A} \int_{0}^{2 \pi} \int_{0}^{1} \delta^{2}(r, \theta) r d r d \theta=\frac{1}{A} \int_{0}^{2 \pi} \int_{0}^{1}\left(\sum_{i=1}^{N} a_{i} Z_{i}(r, \theta)\right)^{2} r d r d \theta \\
& =\frac{1}{A} \int_{0}^{2 \pi} \int_{0}^{1} \sum_{i=1}^{N} a_{i}^{2} Z_{i}^{2}(r, \theta) r d r d \theta=\sum_{i=1}^{N} a_{i}^{2} k_{i}^{2}, \\
\sigma & =\sqrt{\sum_{i=1}^{N} a_{i}^{2} k_{i}^{2}} .
\end{aligned}
$$


TABLE 2: The result of effect of errors distributed in the normalized Zernike polynomials on EMP.

\begin{tabular}{|c|c|c|c|c|c|c|c|c|c|}
\hline \multirow{2}{*}{ Term } & \multicolumn{2}{|c|}{ Loss $(\mathrm{dB})$} & \multicolumn{2}{|c|}{ Offset $\left({ }^{\prime \prime}\right)$} & \multicolumn{4}{|c|}{$\Delta \mathrm{SLD}(\mathrm{dB})$} & \multirow{2}{*}{ Characteristic } \\
\hline & 0 cut & 90 cut & 0 cut & 90 cut & 0 cut-L* & 0 cut- $\mathrm{R}^{*}$ & 90 cut-L & 90 cut- $\mathrm{R}$ & \\
\hline$z 1$ & 0.01 & 0.01 & 0 & 0 & 0.4 & 0.4 & 0.4 & 0.4 & Piston \\
\hline$z 2$ & -0.28 & 0 & 0 & -94.6 & 0.6 & 0.6 & -2.6 & 3.4 & Tilt \\
\hline$z 3$ & 0 & -0.28 & -94.6 & 0 & -2.6 & 3.3 & 0.6 & 0.6 & Tilt \\
\hline$z 4$ & -0.2 & -0.2 & 0 & 0 & -2 & -2 & -2.7 & -2.7 & Astigmatism \\
\hline$z 5$ & -0.44 & -0.44 & 0 & 0 & 6.8 & 6.8 & 6.8 & 6.8 & Defocus \\
\hline$z 6$ & -0.19 & -0.19 & 0 & 0 & 6.1 & 6.1 & 3.8 & 3.8 & Astigmatism \\
\hline$z 7$ & -0.17 & -0.17 & 0 & 0 & 1 & 1 & -7 & 8.3 & \\
\hline$z 8$ & -0.35 & -0.31 & 0 & 40.8 & 2.7 & 2.7 & 10.8 & 0.8 & Coma \\
\hline$z 9$ & -0.29 & -0.33 & 40.8 & 0 & 10.7 & 1.35 & 2.5 & 2.5 & Coma \\
\hline$z 10$ & -0.17 & -0.17 & 0 & 0 & 8 & -2.6 & 0.6 & 0.6 & \\
\hline$z 11$ & -0.16 & -0.16 & 0 & 0 & -0.3 & -0.3 & -0.1 & -0.1 & \\
\hline$z 12$ & -0.29 & -0.29 & 0 & 0 & -2.35 & -2.35 & -2.5 & -2.5 & 2nd-astigm \\
\hline$z 13$ & -0.37 & -0.37 & 0 & 0 & 8.05 & 8.05 & 8.05 & 8.05 & Spherical \\
\hline$z 14$ & -0.28 & -0.28 & 0 & 0 & 8.3 & 8.3 & 9.3 & 9.3 & 2nd-astigm \\
\hline$z 15$ & -0.16 & -0.16 & 0 & 0 & 5 & 5 & 4.6 & 4.6 & \\
\hline$z 16$ & -0.14 & -0.14 & 0 & 0 & 0.55 & 0.55 & 2.9 & 6.4 & \\
\hline$z 17$ & -0.26 & -0.26 & 0 & 0 & 0.3 & 0.3 & 8.8 & 6.7 & \\
\hline$z 18$ & -0.34 & -0.34 & 0 & 0 & 3.0 & 3.0 & 8.8 & 11.6 & 2nd-coma \\
\hline$z 19$ & -0.33 & -0.33 & 0 & 0 & 8.6 & 11.5 & 2.8 & 2.8 & 2nd-coma \\
\hline$z 20$ & -0.26 & -0.26 & 0 & 0 & 7.1 & 9 & -0.8 & -0.8 & \\
\hline$z 21$ & -0.14 & -0.14 & 0 & 0 & -18.8 & 6.2 & 0.8 & 0.8 & \\
\hline
\end{tabular}

*-L means the sidelobe level degradation on the left side; - $\mathrm{R}$ means the sidelobe level degradation on the right side.

The Zernike polynomials can be normalized to ensure that the RMS value of the phase error caused by any polynomial is one. The normalized Zernike polynomials can be described as follows:

$$
Z_{j}^{\prime}(r, \theta)=\frac{Z_{j}(r, \theta)}{k_{j}}
$$

The attractive features of the normalized Zernike polynomial representation are that for each polynomial or Zernike mode (apart from the piston term), the mean value of the aberration across the aperture is zero, and the value of the coefficient gives the RMS wavefront error for that particular Zernike mode.

\section{Effects of Error Distributions}

As mentioned above, the RMS of wavefront errors distributed in a normalized Zernike polynomial is unity; therefore, any wavefront errors distributed in the normalized Zernike polynomials would have the same RMS. Then, it is helpful to study how these errors influence the EMP.

The geometry of a parabolic reflector antenna with a large-scale distortion is depicted in Figure 5. It is assumed that the OPD is described by different normalized Zernike polynomials in the circle aperture. Furthermore, the reflector has a diameter $D=26 \mathrm{~m}$ and a focal length $F=7.8 \mathrm{~m}$. The operation wavelength is $\lambda=0.06 \mathrm{~m}$, and the aperture field is a Gaussian distribution with $-12 \mathrm{~dB}$ edge taper. To obtain the obvious effect, the normalized Zernike polynomials are multiplied by a weight factor ke or in other words the actual distortion RMS. It is assumed $\mathrm{ke}=\lambda / 20=0.3 \mathrm{~cm}$, and the RMS of half of the distortion is $\lambda / 40$. The estimated gain loss with $\mathrm{RMS} / \lambda=1 / 40$ can be calculated by using modified Ruze's formula (equation (1)), and the result is $-0.32 \mathrm{~dB}$. Then, the OPD is modeled by

$$
\delta_{i}(r, \theta)=\mathrm{ke} \cdot \frac{Z_{i}(r, \theta)}{k_{i}}
$$

The far-field patterns with the OPD distributed in the first 21 normalized Zernike polynomials are computed by using the radiation formulation (equation 2). The results of the EMP-gain loss, boresight offset, and sidelobe level degradation-are listed in Table 2, and the far-field patterns in the $0^{\circ}$ and $90^{\circ}$ cut planes, shown in Figure 6, are normalized to the peak of the ideal reflector, without distortions. The beam pattern contours are shown in Figure 7.

The loss item in Table 2 shows that the gain losses are different. Note that the gain losses in $z 5$ and $z 13$ are more 


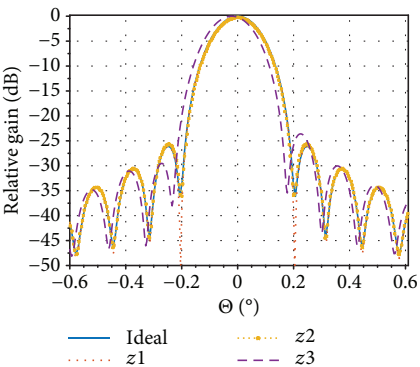

(a1) $0^{\circ}$ cut plane

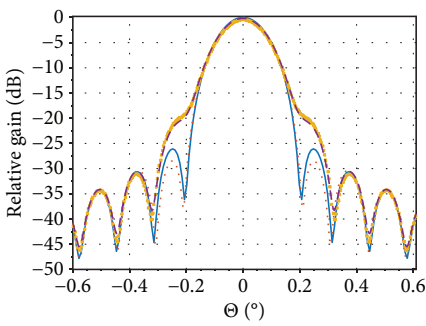

$\begin{array}{lll}\text { Ideal } & \ldots . . \cdots & z 5 \\ \ldots 4 & -. & z 6\end{array}$

(b1) $0^{\circ}$ cut plane

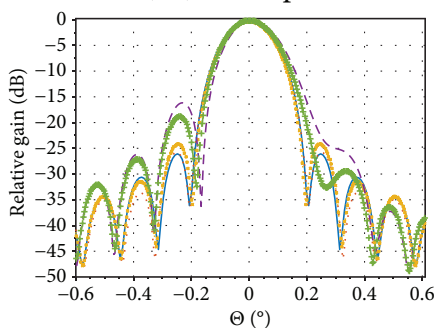

$\begin{array}{lll}\text { Ideal } & --- & z 9 \\ z 7 & -+- & z 10\end{array}$

(c1) $0^{\circ}$ cut plane

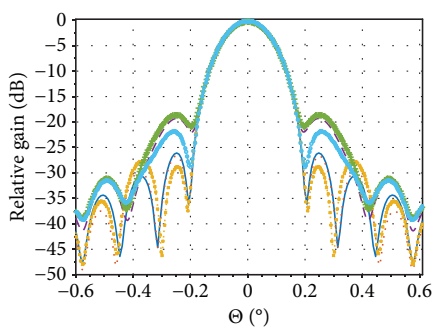

$\begin{array}{lll}\text { Ideal } & --- & z 13 \\ z 11 & -+- & z 14 \\ z 12 & \ldots & z 15\end{array}$

(d1) $0^{\circ}$ cut plane

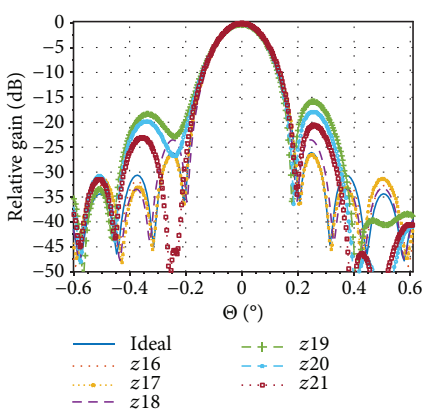

(e1) $0^{\circ}$ cut plane

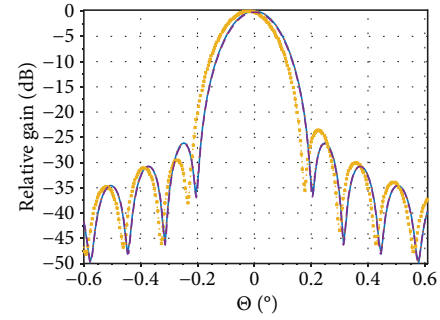

(a2) $90^{\circ}$ cut plane

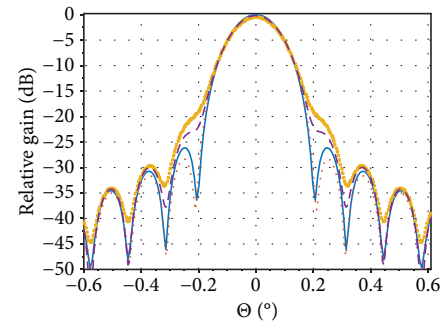

(b2) $90^{\circ}$ cut plane

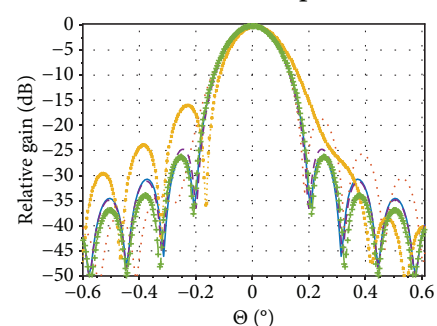

(c2) $90^{\circ}$ cut plane

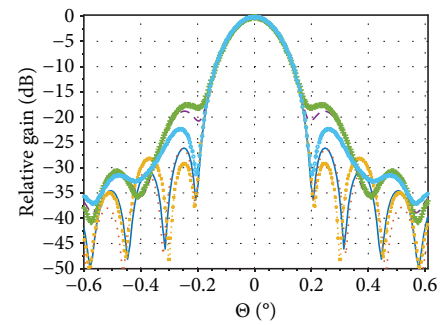

(d2) $90^{\circ}$ cut plane

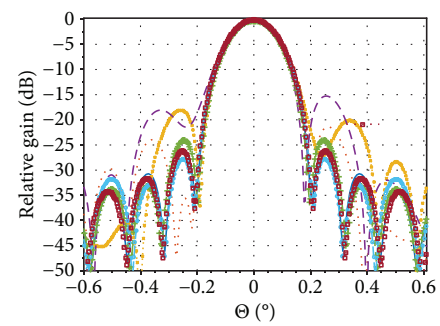

(e2) $90^{\circ}$ cut plane

FIGURE 6: $0^{\circ}$ and $90^{\circ}$ cut plane far-field patterns of the OPD distributed in the first 21 normalized Zernike polynomials. The patterns are normalized to the peak of the ideal pattern. 


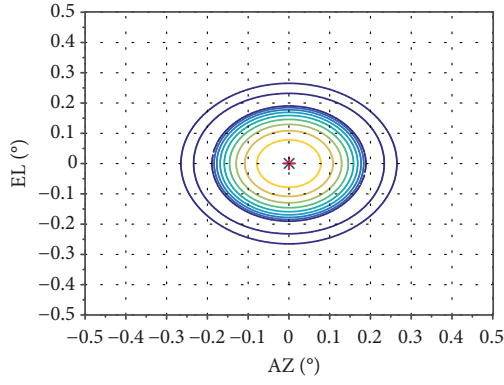

(a) $z 1$

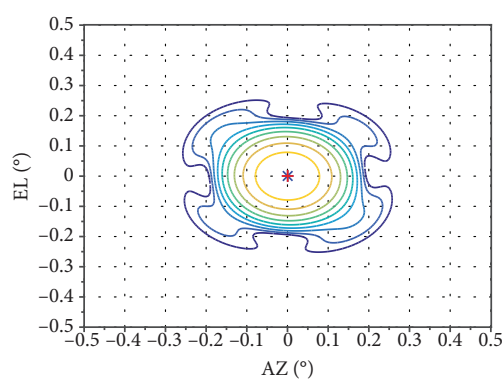

(d) $z 4$

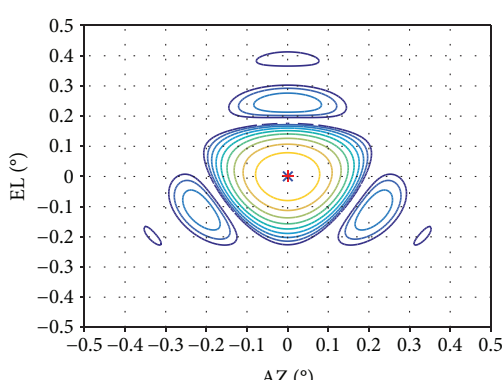

(g) $z 7$

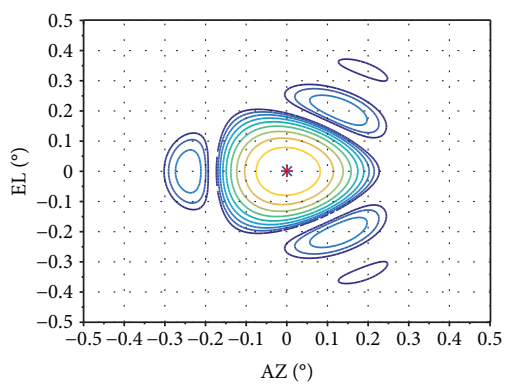

(j) $z 10$

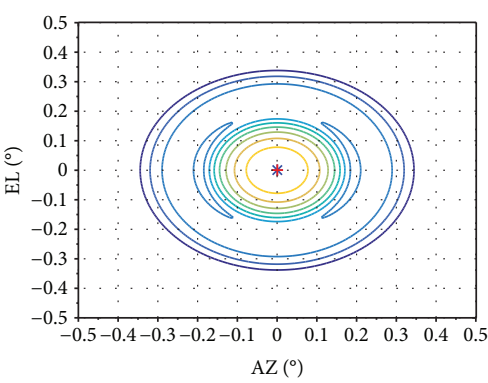

(m) $z 13$

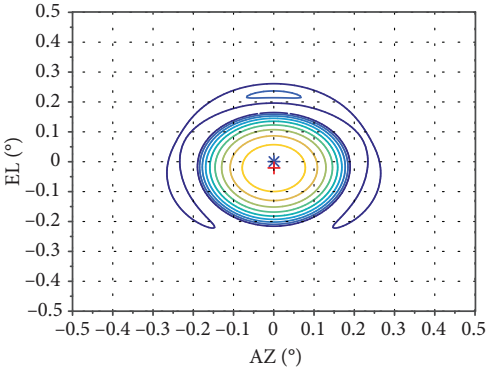

(b) $z 2$

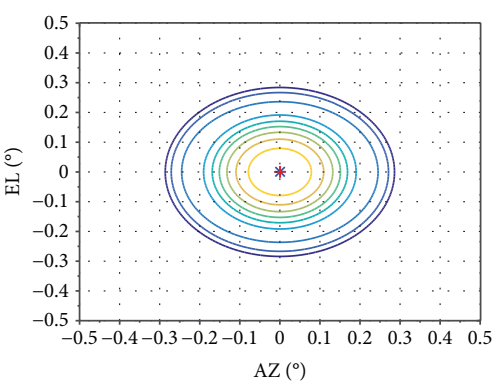

(e) $z 5$

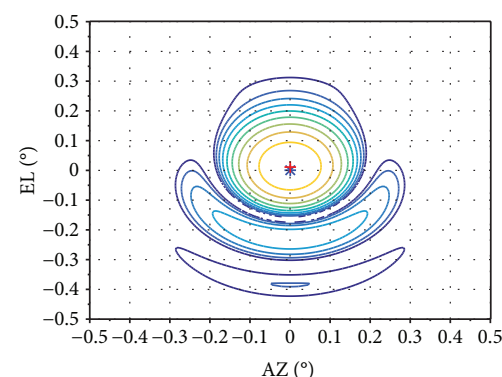

(h) $z 8$

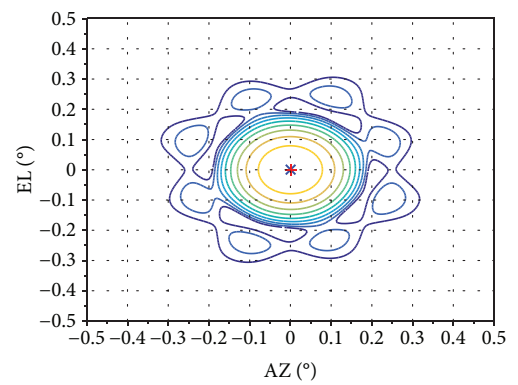

(k) $z 11$

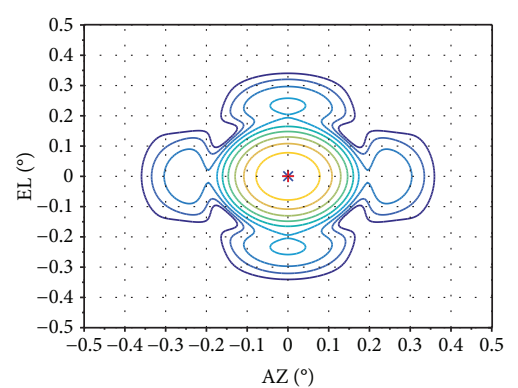

(n) $z 14$

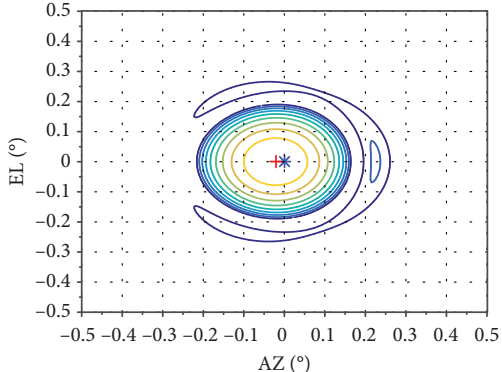

(c) $z 3$

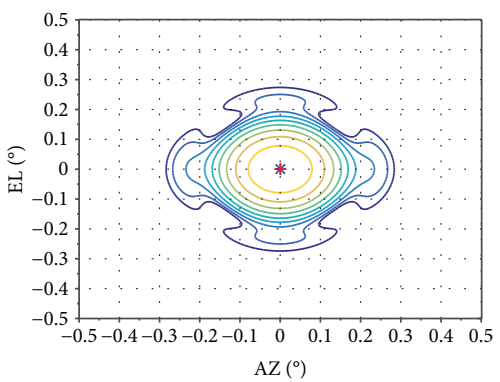

(f) $z 6$

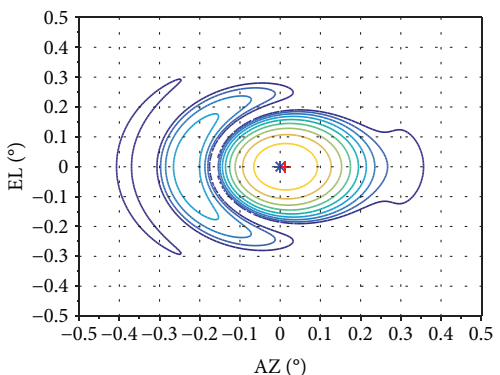

(i) $z 9$

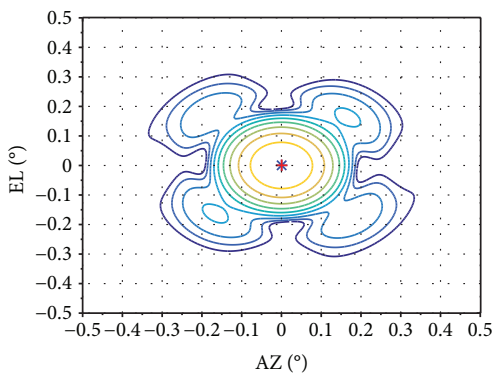

(l) $z 12$

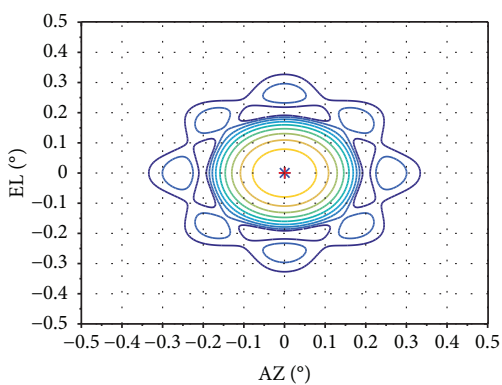

(o) $z 15$

Figure 7: Continued. 


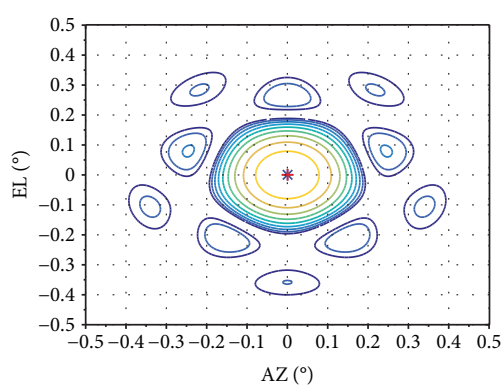

(p) $z 16$

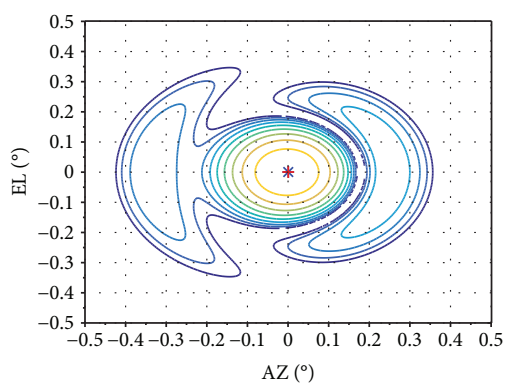

(s) $z 19$

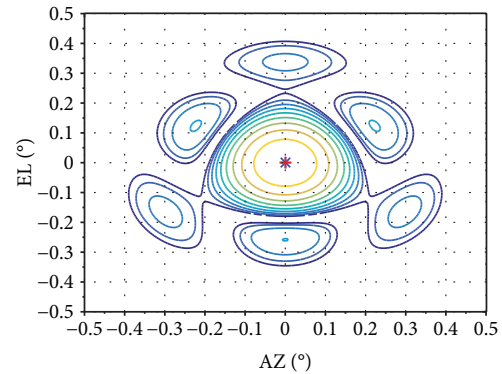

(q) $z 17$

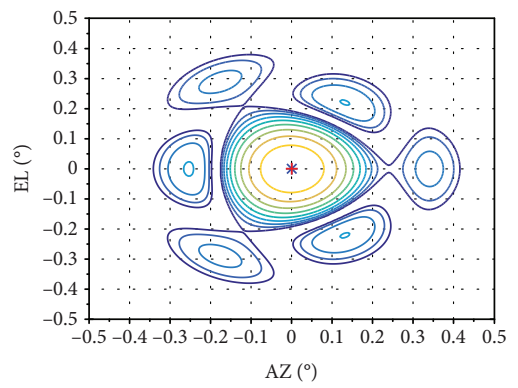

(t) $z 20$

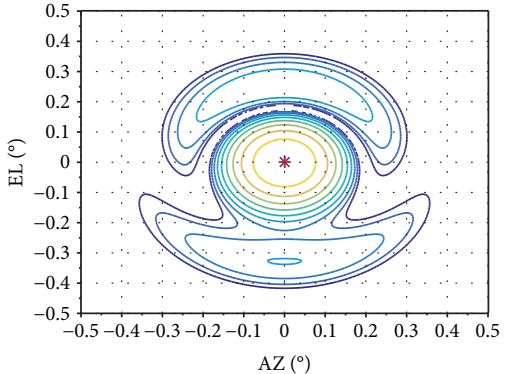

(r) $z 18$

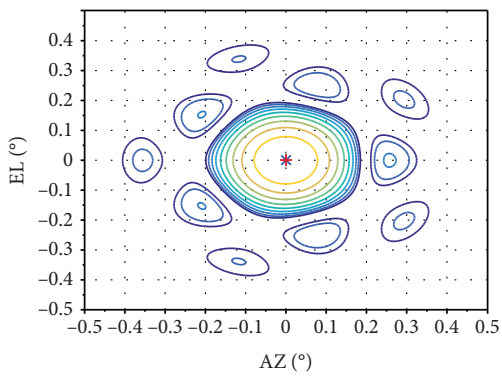

(u) $z 21$

FIGURE 7: Beam contour patterns of the far-field radiation with the OPD distributed in the first 21 normalized Zernike polynomials. The magnitudes of contours range from "peak" to "peak-30 dB."

significant than others. This indicates that the defocus and spherical aberration have a greater impact on the gain.

The offset item in Table 2 shows that $z 2, z 3, z 8$, and $z 9$ have a greater impact on the boresight pointing offsets, and other distributions have almost no effect on the pointing offset. This means that the boresight offset is mainly influenced by tilt and coma.

As shown in Figure 6, almost all the sidelobe levels are increased to different extents. The results of sidelobe level degradation $(\triangle \mathrm{SLD})$ in Table 2 indicate that the sidelobe levels of $z 5$ and $z 13$ are increased to some extent, and these are symmetrical. The sidelobe levels of $z 6$ and $z 14$ are increased to some extent, and these are asymmetrical; it means that their sidelobe levels in $0^{\circ}$ and $90^{\circ}$ cut planes are different, but the sidelobe levels in the same cut plane are equal. The peak levels of sidelobe degradation of $z 8, z 9$, $z 18$, and $z 19$ are fairly high, and their levels of left and right sidelobes in the $0^{\circ}$ or $90^{\circ}$ cut plane are different; it means that one side is high and the other one is low. From the distribution characteristic, except the characteristics of defocus and spherical aberration (excluding piston), the other characteristics will lead to sidelobe level asymmetry. From the overall results of Table 2, we can see that the greatest influences on sidelobe levels are the coma (the peak of $\triangle \mathrm{SLD}$ is about $10.7 \mathrm{~dB}$ ) and 2nd-coma (the peak of $\triangle \mathrm{SLD}$ is about $11.6 \mathrm{~dB}$ ), followed by 2 nd-astigmatism (the peak of $\triangle \mathrm{SLD}$ is about $9.3 \mathrm{~dB}$ ) and spherical aberration (the peak of $\triangle \mathrm{SLD}$ is about $8 \mathrm{~dB}$ ); next are defocus (the peak of $\triangle \mathrm{SLD}$ is about $6.8 \mathrm{~dB}$ ) and astigmatism (the peak of $\triangle \mathrm{SLD}$ is about $6.1 \mathrm{~dB}$ ), and the tilt has the least influence (the peak of $\triangle \mathrm{SLD}$ is about $3.4 \mathrm{~dB}$ ).

The beam contour patterns of the far-field radiation are shown in Figure 7. Patterns are plotted in ranges of magnitudes from "peak" to "peak-30 dB," and the magnitude difference of the adjacent contours is $3 \mathrm{~dB}$. We can see in Figure 7 that the error distribution forms have a significant effect on pattern shapes and cause different distortions of pattern shapes. The shapes of patterns with the same aberration type are similar, such as the patterns caused by $z 2$ and that caused by $z 3$.

The results are shown in Figures 5 and 6, and we can know that the gain losses, sidelobe levels, and pattern shapes caused by wavefront errors with the same RMS value are different and are distributed with different distribution characteristics. It shows that the beam patterns are correlated with the distribution forms of distortions. Thus, the coefficient of Zernike polynomials can be applied in modeling the wavefront shapes to decompose the wavefront aberrations.

\section{Effects of Aberrations}

To further investigate the effect of the Zernike coefficient on EMP, the distributions of wavefront errors are analyzed from the optical aberration point of view. The Zernike decomposition provides a good connection between EMP and the wavefront error distribution, which represents the aberration component [19]. Each polynomial function of the Zernike expansion extracts a component of the wavefront error; these components can be grouped into classical aberrations called Seidel aberration.

According to the Seidel aberration theory, the first-order wavefront properties and third-order wavefront aberration coefficients can be obtained from the Zernike polynomial coefficients [17]. The nine Zernike polynomials are adopted to describe the Seidel aberration. The aberrations and properties corresponding to these Zernike terms are shown in 
Table 3. Using the first nine new Zernike terms $z 0^{\prime}$ to $z 8^{\prime}$ in Table 3, the wavefront errors can be written as the combination of the first nine new Zernike terms as follows:

$$
\begin{aligned}
\delta(r, \theta)= & W(r, \theta)=\sum_{i=0}^{8} a_{i} Z_{i}^{\prime}(r, \theta) \\
= & a_{0}+a_{1} r \cos \theta+a_{2} r \sin \theta+a_{3}\left(2 r^{2}-1\right) \\
& +a_{4} r^{2} \cos 2 \theta+a_{5} r^{2} \sin 2 \theta+a_{6}\left(3 r^{3}-2 r\right) \cos \theta \\
& +a_{7}\left(3 r^{3}-2 r\right) \sin \theta+a_{8}\left(6 r^{4}-6 r^{2}+1\right),
\end{aligned}
$$

where $a_{i}$ represents the new Zernike polynomial coefficients. Rewriting the new Zernike expansion of equation (15), the first- and third-order field-independent wavefront aberration terms can be obtained. This is achieved by grouping the similar terms and equating them with the wavefront aberration coefficients:

$$
\begin{aligned}
& W(r, \theta)=a_{0}-a_{3}+a_{8} \quad \text { piston } \\
& +\left(a_{1}-2 a_{6}\right) r \cos \theta+\left(a_{2}-2 a_{7}\right) r \sin \theta \quad \text { tilt } \\
& +\left(2 a_{3}-6 a_{8}\right) r^{2} \\
& +a_{4} r^{2} \cos 2 \theta+a_{5} r^{2} \sin 2 \theta \\
& +3 a_{6} r^{3} \cos \theta+3 a_{7} r^{3} \sin \theta \\
& +6 a_{8} r^{4}
\end{aligned}
$$

This equation can be rearranged corresponding to the field-independent wavefront aberration coefficients as follows:

$$
\begin{aligned}
& W(r, \theta)=a_{0}-a_{3}+a_{8} \\
& +\sqrt{\left(a_{1}-2 a_{6}\right)^{2}+\left(a_{2}-2 a_{7}\right)^{2}} \cdot r \\
& \times \cos \left[\theta-\tan ^{-1}\left(\frac{a_{2}-2 a_{7}}{a_{1}-2 a_{6}}\right)\right] \\
& +\left(2 a_{3}-6 a_{8} \pm \sqrt{a_{4}^{2}+a_{5}^{2}}\right) \cdot r^{2} \\
& \pm 2 \sqrt{a_{4}^{2}+a_{5}^{2}} \cdot r^{2} \cos ^{2}\left[\theta-\frac{1}{2} \tan ^{-1}\left(\frac{a_{5}}{a_{4}}\right)\right] \\
& +3 \sqrt{a_{6}^{2}+a_{7}^{2}} \cdot r^{3} \cos \left[\theta-\tan ^{-1}\left(\frac{a_{7}}{a_{6}}\right)\right] \quad \text { coma } \\
& +6 a_{8} \cdot r^{4}
\end{aligned}
$$

From equation (17), the magnitude, sign, and angle of these field-independent aberration terms are listed in Table 4, and the corresponding shapes of the Seidel wavefront aberration are shown in Figure 8.
TABLE 3: The nine Zernike terms and aberrations.

\begin{tabular}{lccc}
\hline $\begin{array}{l}\text { New } \\
\text { terms }\end{array}$ & $\begin{array}{c}\text { Zernike } \\
\text { terms }\end{array}$ & Polynomials & Characteristic \\
\hline$z 0^{\prime}$ & $z 1$ & 1 & Piston \\
$z 1^{\prime}$ & $z 3$ & $r \cos \theta$ & $x$-tilt \\
$z 2^{\prime}$ & $z 2$ & $r \sin \theta$ & $y$-tilt \\
$z 3^{\prime}$ & $z 5$ & $2 r^{2}-1$ & Defocus \\
$z 4^{\prime}$ & $z 6$ & $r^{2} \cos 2 \theta$ & Horizontal astigmatism \\
$z 5^{\prime}$ & $z 4$ & $r^{2} \sin 2 \theta$ & Oblique astigmatism \\
$z 6^{\prime}$ & $z 9$ & $\left(3 r^{3}-2 r\right) \cos \theta$ & $x$-coma \\
$z 7^{\prime}$ & $z 8$ & $\left(3 r^{3}-2 r\right) \sin \theta$ & $y$-coma \\
$z 8^{\prime}$ & $z 13$ & $6 r^{4}-6 r^{2}+1$ & Spherical \\
\hline
\end{tabular}

As mentioned above, the effects of the normalized Seidel wavefront aberrations on EMP - which are multiplied by a weight factor (1/RMS) - are computed. In the analysis, the reference angles of tilt, astigmatism, and coma are set to zero. The results of EMP are listed in Table 5, and the far-field patterns in the $0^{\circ}$ and $90^{\circ}$ cut planes are shown in Figure 9 and are normalized to the peak of the ideal reflector. The beam contour patterns are shown in Figure 10.

The result of Table 5 indicates that the terms $s 2, s 3$, and $s 5$ have a greater impact on gain loss; the terms $s 1$ and $s 4$ mainly have effect on the pointing offset and, moreover, cause the asymmetric sidelobes in the cut planes.

Based on many numerical simulations, the relationships between gain loss $\Delta G$ and the different aberration coefficients $a$ have been determined and are shown in Figure 11. The relationship can be given by

$$
\Delta G=h \cdot a^{\prime 2}
$$

where $h$ is the gain loss influence factor and $a^{\prime}$ is the aberration coefficient normalized to the wavelength, $a / \lambda$. Through simulations, the gain loss influence factors for the focus, astigmatism, and spherical aberration have be obtained, and the values of $h$ are $-8.777,-3.657$, and -7.132 , respectively.

The relationships between the aberration coefficients and the boresight pointing offsets have been determined and are shown in Figure 12 and can be expressed as follows:

$$
\begin{aligned}
& \Delta P_{t}=k_{t} \cdot a, \\
& \Delta P_{c}=k_{c 1} \cdot a^{2}+k_{c 2} \cdot a,
\end{aligned}
$$

where $\Delta P_{t}$ and $\Delta P_{c}$ are the boresight pointing offsets caused by tilt and coma, respectively. $k_{t}, k_{c 1}$, and $k_{c 2}$ are the corresponding pointing offset influence factors; $k_{t}=-3.526, k_{c 1}=$ 3.94 , and $k_{c 2}=-2.2$.

Similarly, the relationships between the normalized aberration coefficients and the sidelobe level increase ( $\Delta$ SLL) have been determined and are shown in Figure 13, and they 
TABLE 4: The Seidel aberration in terms of Zernike coefficients.

\begin{tabular}{|c|c|c|c|c|}
\hline Aberration & Polynomials & Magnitude & Angle $\theta^{\prime}$ & RMS \\
\hline Tilt & $s 1=r \cos \left(\theta-\theta^{\prime}\right)$ & $\sqrt{\left(a_{1}-2 a_{6}\right)^{2}+\left(a_{2}-2 a_{7}\right)^{2}}$ & $\tan ^{-1}\left(a_{2}-2 a_{7} / a_{1}-2 a_{6}\right)$ & $\frac{1}{2}$ \\
\hline Focus & $s 2=r^{2}$ & $2 a_{3}-6 a_{8} \pm \sqrt{a_{4}^{2}+a_{5}^{2}}$ & & $\frac{1}{\sqrt{3}}$ \\
\hline Astigmatism & $s 3=r^{2} \cos ^{2}\left(\theta-\theta^{\prime}\right)$ & $\pm 2 \sqrt{a_{4}^{2}+a_{5}^{2}}$ & $1 / 2 \tan ^{-1}\left(a_{5} / a_{4}\right)$ & $\frac{1}{\sqrt{8}}$ \\
\hline Coma & $s 4=r^{3} \cos \left(\theta-\theta^{\prime}\right)$ & $3 \sqrt{a_{6}^{2}+a_{7}^{2}}$ & $\tan ^{-1}\left(a_{7} / a_{6}\right)$ & $\frac{1}{\sqrt{8}}$ \\
\hline Spherical & $s 5=r^{4}$ & $6 a_{8}$ & & $\frac{1}{\sqrt{5}}$ \\
\hline
\end{tabular}

Note: the sign of the magnitude of the focus term is chosen to minimize the magnitude of the coefficient, and astigmatism uses the sign opposite that.

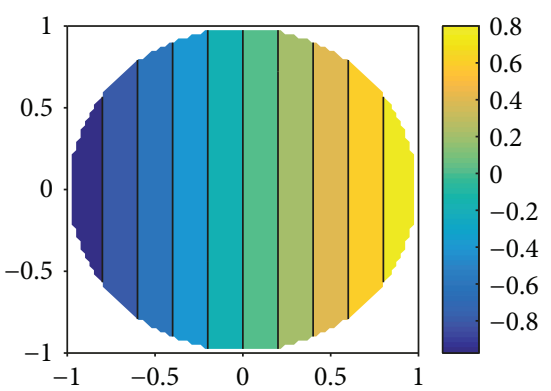

(a) $s 1$

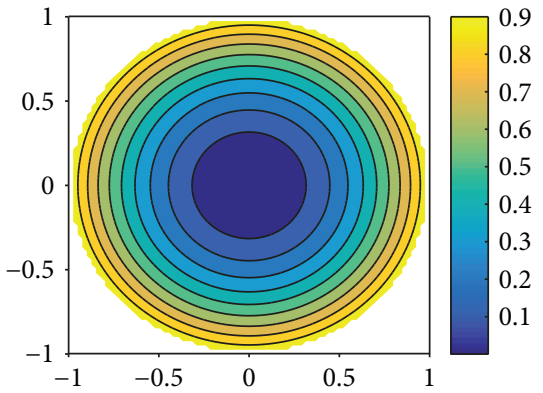

(b) $s 2$

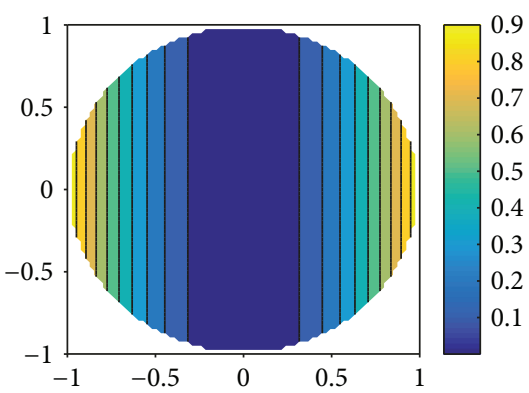

(c) $s 3$

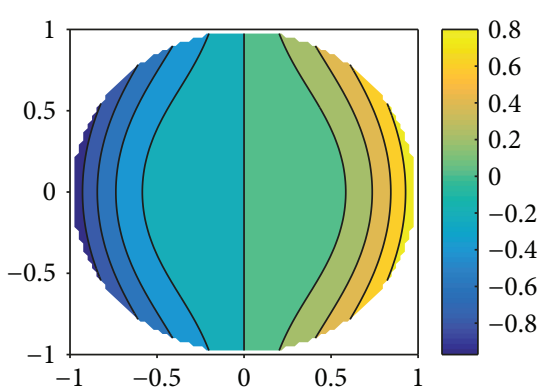

(d) $s 4$

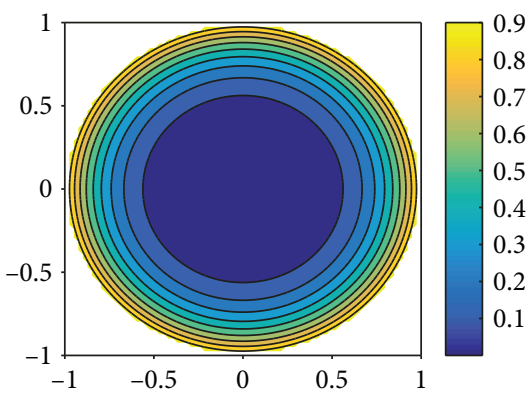

(e) $s 5$

Figure 8: Shape characteristics of the Seidel wavefront aberration.

TABLE 5: The result of effect of the normalized Seidel wavefront aberrations on EMP.

\begin{tabular}{|c|c|c|c|c|c|c|c|c|c|}
\hline \multirow{2}{*}{ Seidel term } & \multicolumn{2}{|c|}{ Loss $(\mathrm{dB})$} & \multicolumn{2}{|c|}{ Offset $\left(^{\prime \prime}\right)$} & \multicolumn{4}{|c|}{$\Delta$ SLD (dB) } & \multirow{2}{*}{ Aberration } \\
\hline & 0 cut & 90 cut & 0 cut & 90 cut & 0 cut-L & 0 cut- $\mathrm{R}$ & 90 cut-L & 90 cut-R & \\
\hline$s 1$ & 0 & -0.37 & -95.2 & 0 & -4.2 & 4 & 0.4 & 0.4 & Tilt \\
\hline$s 2$ & -0.19 & -0.19 & 0 & 0 & 2.4 & 2.4 & 4.3 & 4.3 & Focus \\
\hline$s 3$ & -0.19 & -0.19 & 0 & 0 & 7 & 7 & 0.6 & 0.6 & Astigmatism \\
\hline$s 4$ & -0.03 & -0.25 & -81.6 & 0 & 4.6 & -7.4 & -0.2 & -0.2 & Coma \\
\hline$s 5$ & -0.23 & -0.23 & 0 & 0 & 1.9 & 1.9 & 2.6 & 2.6 & Spherical \\
\hline
\end{tabular}

can be expressed by

$$
\Delta \mathrm{SLL}=\mathrm{ks}_{a} \cdot a^{\prime 2}+\mathrm{ks}_{b} \cdot a^{\prime},
$$

where $\mathrm{ks}_{a}$ and $\mathrm{ks}_{b}$ are the sidelobe level influence coefficients, which are different for different aberrations. The values of $\mathrm{ks}_{a}$ for tilt, focus, astigmatism, coma, and spherical aberration 


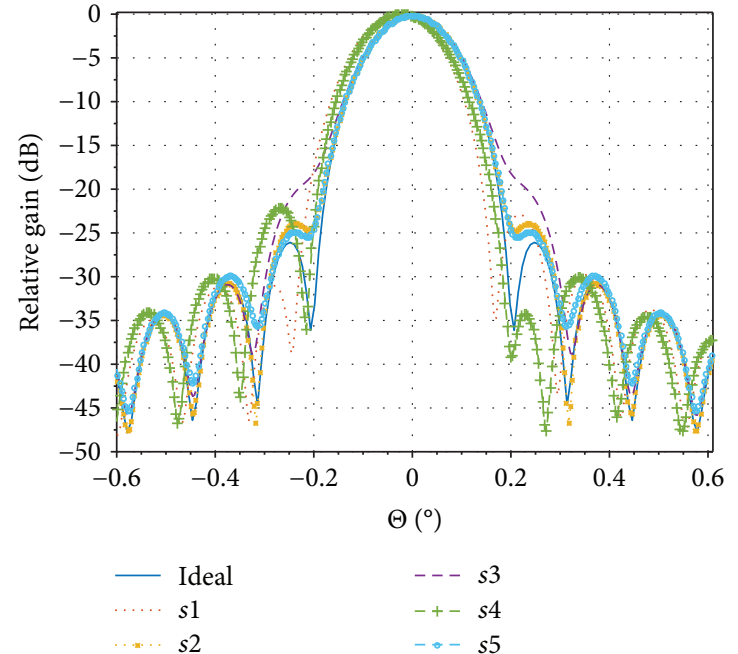

(a) $0^{\circ}$ cut plane

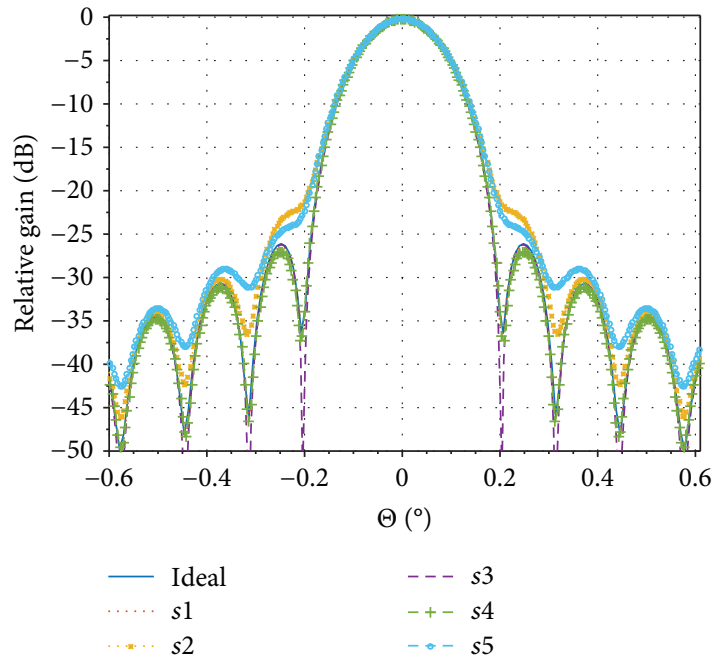

(b) $90^{\circ}$ cut plane

FIGURE 9: $0^{\circ}$ and $90^{\circ}$ cut plane far-field patterns of the normalized Seidel wavefront aberration. The patterns are normalized to the peak of the ideal pattern.

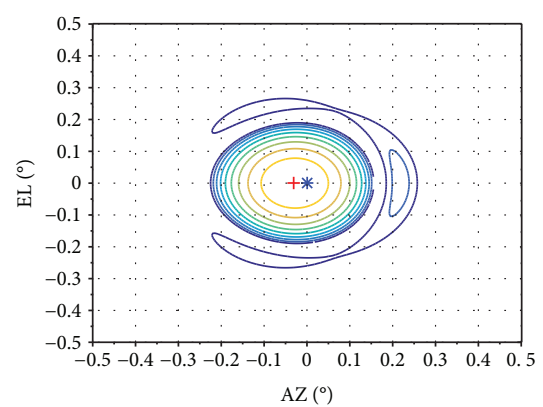

(a) $s 1$

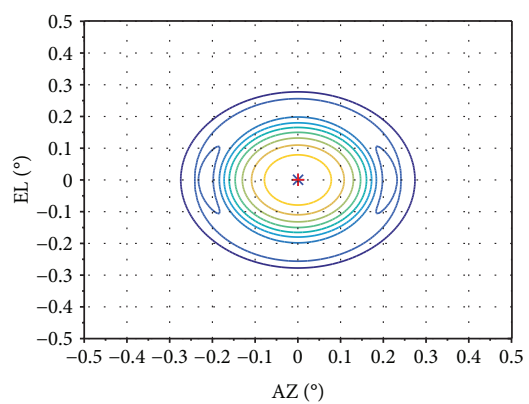

(b) $s 2$

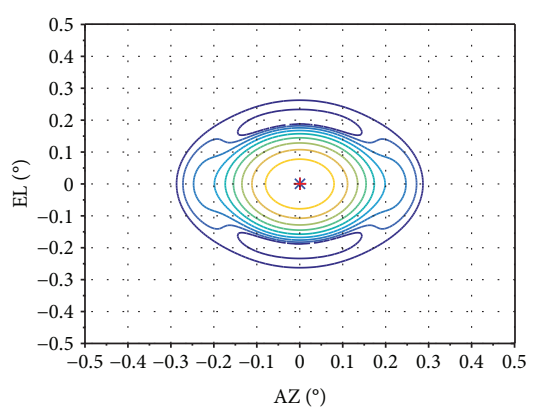

(c) $s 3$

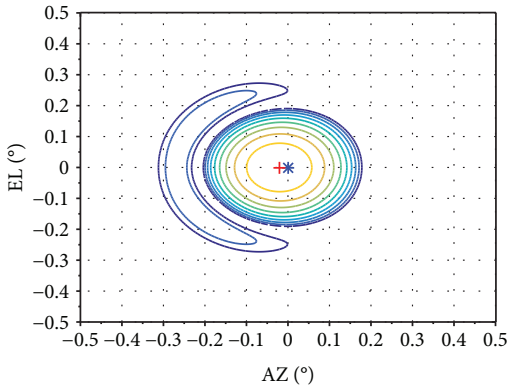

(d) $s 4$

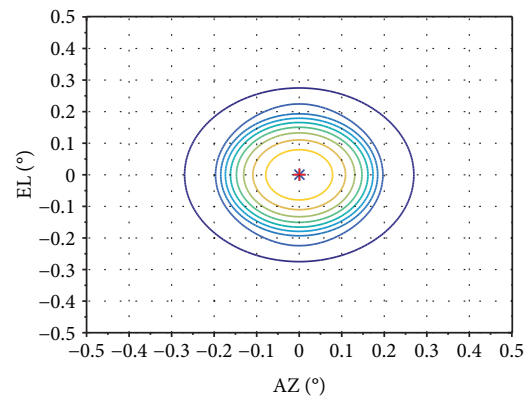

(e) $s 5$

Figure 10: The beam contour patterns of the normalized Seidel wavefront aberration. The magnitudes of contours range from "peak" to "peak-30 dB."

are $-25.6,-48.46,-67.66,-18.29$, and 1.92 , and that of $\mathrm{ks}_{b}$ are $27.2,54.25,58.8,23.18$, and 13.0 , respectively.

So far, the effects of wavefront errors with the aberration forms have been studied. The arbitrary wavefront error can be expressed by the Zernike expansions, and the Seidel aberration coefficient can be computed by using equation (17) and Table 4. Based on the aberration coefficients, the weight of the effects of different types of aberrations can be determined. Thus, the aberration coefficients can serve as a useful metric to evaluate the wavefront distortion behavior and to determine the primary feature of the influence of distortion.

\section{Conclusion}

To understand the effects of arbitrary wavefront errors, it is necessary to investigate the effects of different distribution characteristics of surface distortions on EMP. On the basis of the correlation interval of error distribution, the surface 


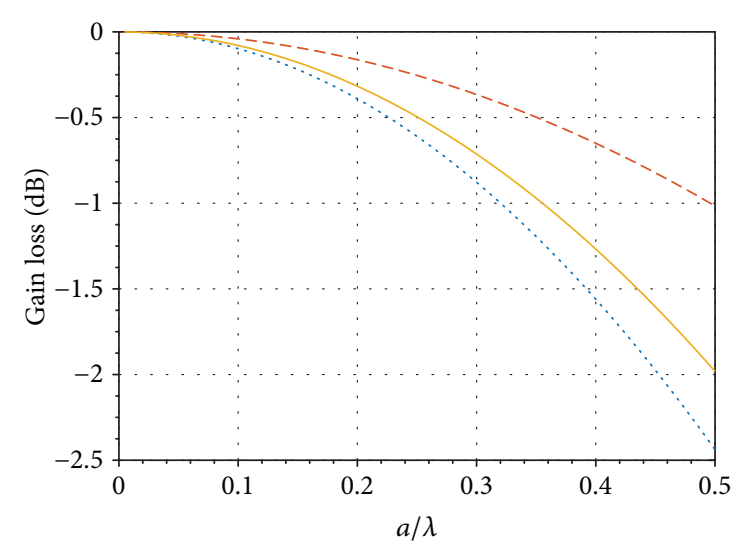

$\cdots$ Focus

- - Astigmatism

- Spherical

Figure 11: Gain loss $\Delta G$ as a function of $a / \lambda$.

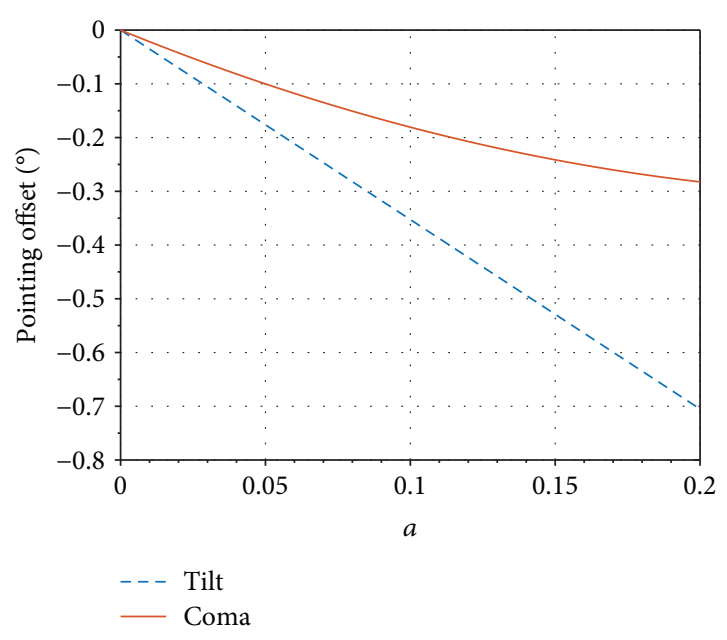

Figure 12: Pointing offset $\Delta P$ as a function of $a$.

distortions are divided into small-scale, medium-scale, and large-scale errors. We proposed that the large-scale errors can be described by the Zernike polynomials. The analysis indicated that distortions in the distribution features of the defocus and the spherical aberration have a greater impact on the gain, and the distribution features of the tilt and the coma mainly influence the boresight offset; the distribution features of the defocus, astigmatism, and spherical aberration have a greater impact on sidelobe levels. Besides, the beam patterns show the similarity between different beam shapes with the same distribution forms of distortions. Based on the Seidel aberration, the relationships between typical EMPs and the aberration coefficients are presented. The gain loss is proportional to the square of the aberration coefficients of the focus, the astigmatism, and the spherical aberration. The boresight pointing offset is proportional to the tilt aberration coefficient, and the relationship between the aberration coefficient and the boresight pointing offset of the coma is a quadratic polynomial. The relationships between the

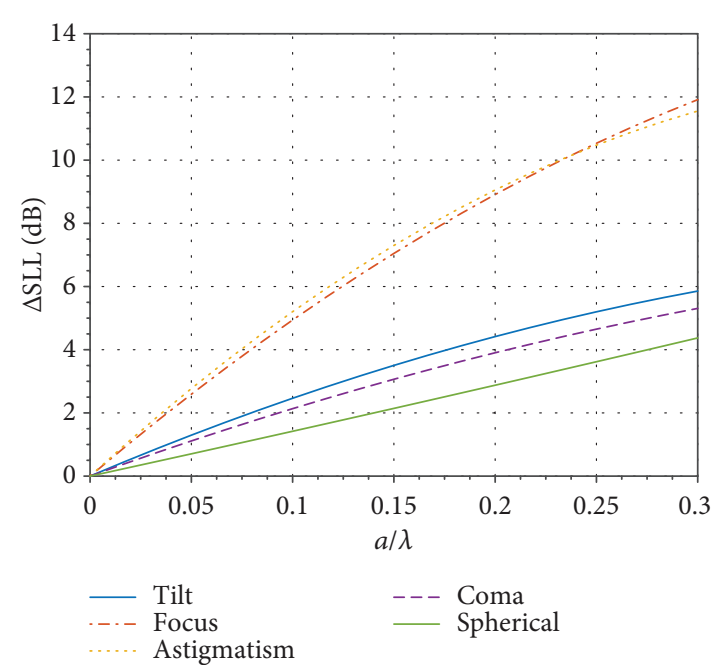

FIGURE 13: Sidelobe level increase $\Delta$ SLL as a function of $a / \lambda$.

normalized aberration coefficients and the sidelobe level increase are also a quadratic polynomial. We can predict the antenna performance with the help of the aberration coefficients. Therefore, the aberration coefficient can be considered as a metric to evaluate the wavefront distortion behavior and to determine the primary influence feature of distortions.

\section{Data Availability}

All the figure data created during this research have been deposited in the Figshare data archive repository (10.6084/ m9.figshare.7229108.v1). We have annotated the entire data substances and data types in "readme.txt."

\section{Conflicts of Interest}

The authors declare that they have no conflicts of interest.

\section{Acknowledgments}

This work was supported by the National Key Basic Research Program of China (grant number 2015CB857100), the Chinese Academy of Sciences (CAS) "Light of West China” Program (grant number 2017-XBQNXZ-B-021), the National Natural Science Foundation of China (grant numbers 51805399 and U1737211), and the Natural Science Foundation of Shaanxi Province (grant number 2018JZ5001). The work was also partly supported by the Operation, Maintenance, and Upgrading Fund for Astronomical Telescopes and Facility Instruments, budgeted from the Ministry of Finance of China (MOF) and administrated by CAS.

\section{References}

[1] Y. Rahmat-Samii and A. Densmore, "A history of reflector antenna development: past, present and future," in 2009 SBMO/IEEE MTT-S International Microwave and Optoelectronics Conference (IMOC), pp. 17-23, Belem, Brazil, November 2010 . 
[2] R. L. Haupt and Y. Rahmat-Samii, "Antenna array developments: a perspective on the past, present and future," IEEE Antennas \& Propagation Magazine, vol. 57, no. 1, pp. 8696, 2015.

[3] J. W. M. Baars, A. Greve, H. Hein, D. Morris, J. Penalver, and C. Thum, "Design parameters and measured performance of the IRAM 30-m millimeter radio telescope," Proceedings of the IEEE, vol. 82, no. 5, pp. 687-696, 1994.

[4] H. J. Kaercher, "Telescopes as mechatronic systems," IEEE Antennas and Propagation Magazine, vol. 48, no. 2, pp. 1737, 2006.

[5] A. Greve, M. Dan, and J. Penalver, "Thermal behavior of millimeter wavelength radio telescopes," IEEE Transactions on Antennas \& Propagation, vol. 40, no. 11, pp. 13751388, 1992.

[6] A. Greve and H. J. Ü. Karcher, "Performance improvement of a flexible telescope through metrology and active control," Proceedings of the IEEE, vol. 97, no. 8, pp. 1412-1420, 2009.

[7] L. Hao, Y. Lo, and Y. Rahmat-Samii, "Reflector sidelobe degradation due to random surface errors," IEEE Transactions on Antennas and Propagation, vol. 34, no. 2, pp. 164-172, 1986.

[8] J. Ruze, “Antenna tolerance theory-a review," Proceedings of the IEEE, vol. 54, no. 4, pp. 633-640, 1966.

[9] Y. Rahmat-Samii, "An efficient computational method for characterizing the effects of random surface errors on the average power pattern of reflectors," IEEE Transactions on Antennas and Propagation, vol. 31, no. 1, pp. 92-98, 1983.

[10] R. P. Corkish, "A survey of the effects of reflector surface distortions on sidelobe levels," IEEE Antennas and Propagation Magazine, vol. 32, no. 6, pp. 6-11, 1990.

[11] K. Bahadori and Y. Rahmat-Samii, "Characterization of effects of periodic and aperiodic surface distortions on membrane reflector antennas," IEEE Transactions on Antennas and Propagation, vol. 53, no. 9, pp. 2782-2791, 2005.

[12] B. Y. Duan and C. S. Wang, "Reflector antenna distortion analysis using MEFCM," IEEE Transactions on Antennas and Propagation, vol. 57, no. 10, pp. 3409-3413, 2009.

[13] P. Y. Lian, B. Y. Duan, W. Wang, B. Xiang, and N. Hu, "Effects of nonuniform surface errors along the radius on reflector's radiation characteristic and its quality evaluation," IEEE Transactions on Antennas and Propagation, vol. 63, no. 5, pp. 2312-2316, 2015.

[14] A. Greve, C. Kramer, and W. Wild, "The beam pattern of the IRAM 30-m telescope (a reflector with several surface error distributions)," Astronomy and Astrophysics Supplement Series, vol. 133, no. 2, pp. 271-284, 1998.

[15] R. Levy, Structural Engineering of Microwave Antennas for Electrical, Mechanical, and Civil Engineers, IEEE Press, 1996.

[16] R. J. Noll, "Zernike polynomials and atmospheric turbulence," Journal of the Optical Society of America, vol. 66, no. 3, pp. 207-211, 1976.

[17] J. C. Wyant and K. Creath, "Basic wavefront aberration theory for optical metrology," Applied optics and optical engineering, vol. 11, no. s29, p. 2, 1992.

[18] V. N. Mahajan, "Zernike circle polynomials and optical aberrations of systems with circular pupils," Applied Optics, vol. 33, no. 34, pp. 8121-8124, 1994.

[19] W. T. Welford, Aberrations of Optical Systems, CRC Press, 1986. 


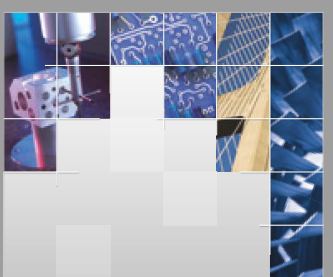

\section{Enfincering}
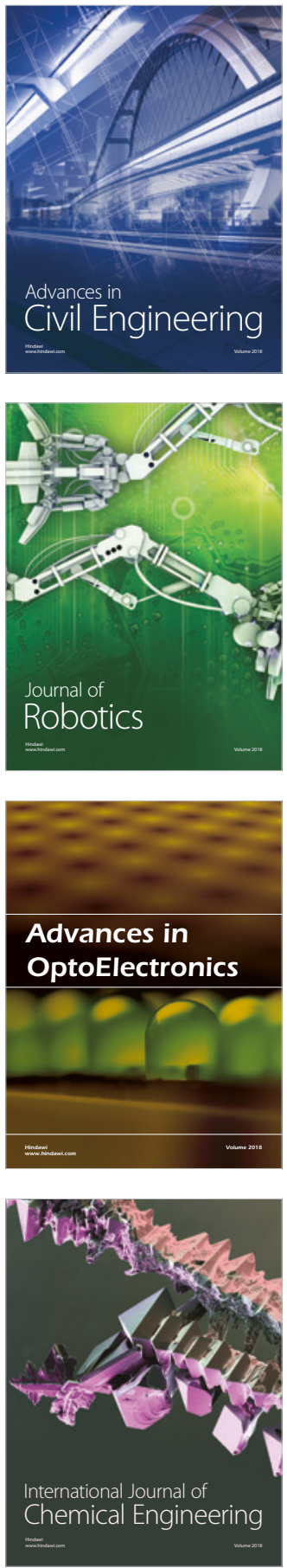

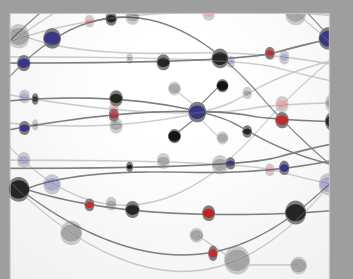

\section{Rotating \\ Machinery}

The Scientific World Journal

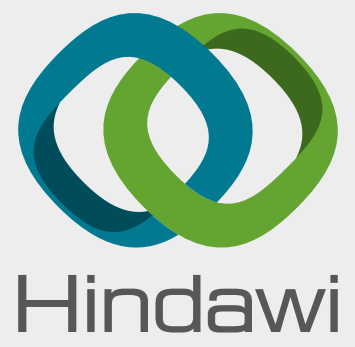

Submit your manuscripts at

www.hindawi.com
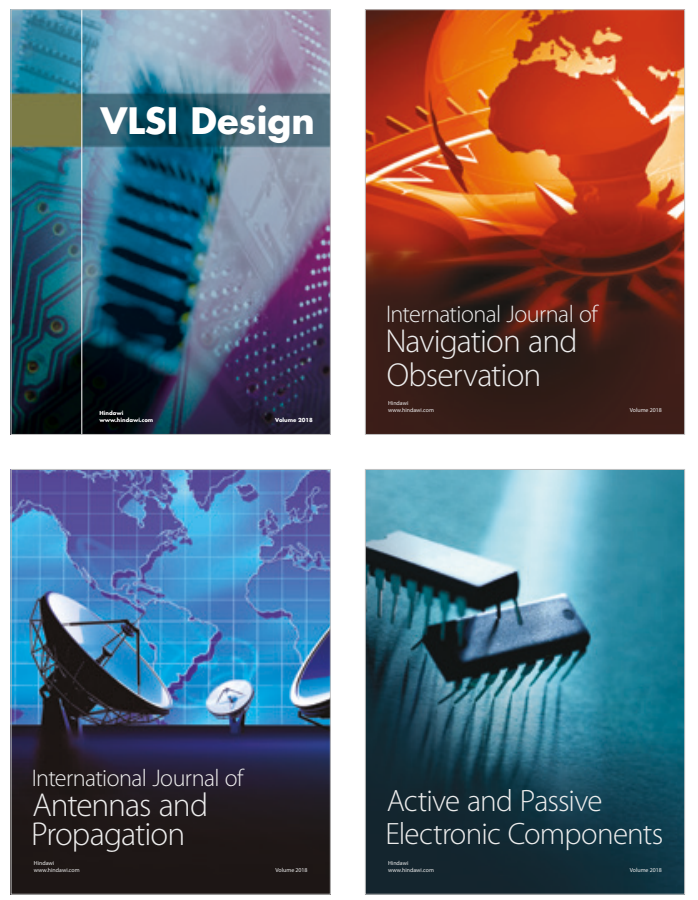
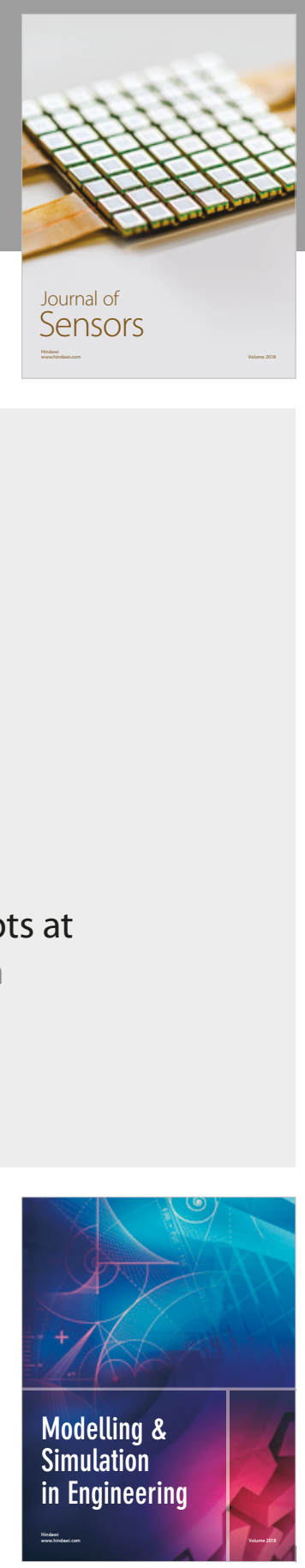

\section{Advances \\ Multimedia}
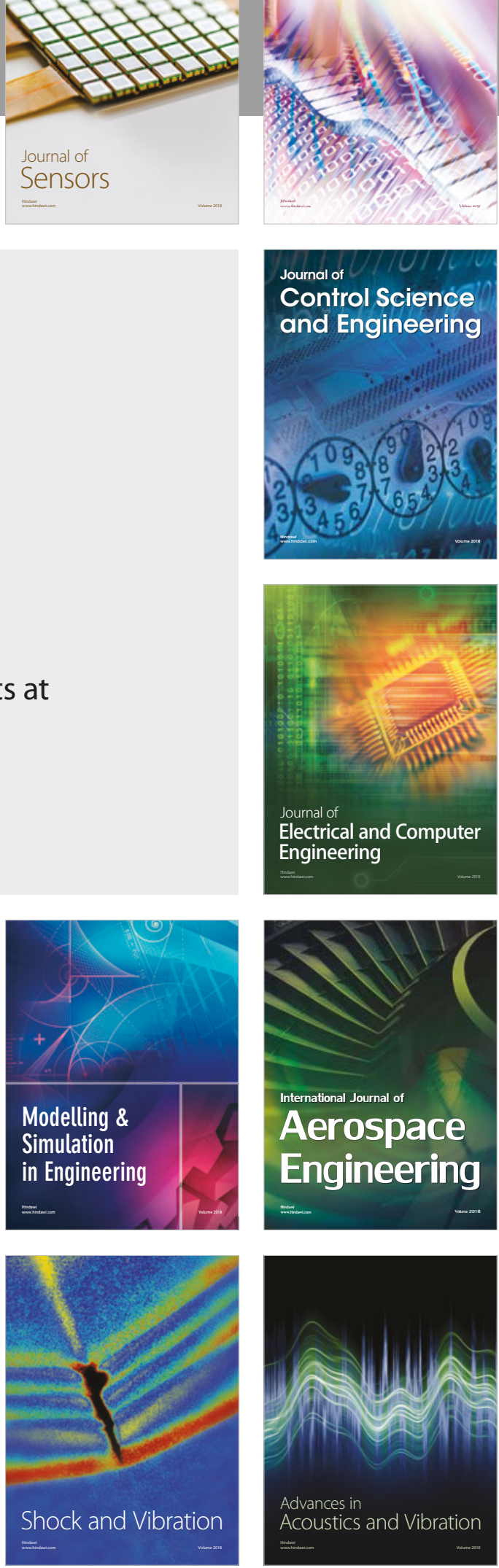